\title{
Distribution et usages des Ampelocissus dans la zone de Donia au sud du Tchad
}

\author{
Mberdoum Memti NGUINAMBAYE ${ }^{1 *}$, Rasmata NANA ${ }^{1}$, Elisée MBAYNGONE ${ }^{2}$, \\ Alain Ignassou DJINET ${ }^{1}$, Badoua BADIEL ${ }^{1}$ et Zoumbiéssé TAMINI ${ }^{1}$ \\ ${ }^{1}$ Laboratoire de Biosciences, Université de Ouagadougou, 03 BP 7023 Ouagadougou 03, Burkina Faso. \\ ${ }^{2}$ Laboratoire de Botanique Systématique et Ecologie Végétale, Université de Ndjamena BP 1027, Tchad. \\ *Auteur correspondant,E-mail:memti2012@hotmail.fr
}

\section{RESUME}

Cette étude a consisté à faire l'inventaire des espèces du genre Ampelocissus à Donia dans le sud du Tchad, à déterminer leur distribution, leurs usages et de caractériser la flore ligneuse. Des prospections ont été faites et à la suite, des placettes ont été disposées dans les sites d'occurrence du taxon pour des relevés floristiques. A cet effet, 110 relevés phytosociologiques ont été réalisés et a permis de déterminer la répartition des espèces suivant leur préférence édaphique. Un herbier a été confectionné et par comparaison aux spécimens existants, une seule espèce du genre Ampelocissus a été identifiée notamment Ampelocissus multistriata (Baker) Planch. Les usages de cette espèce ont été également déterminés à travers des enquêtes. Les résultats ont révélé que Ampelocissus multistriata intervient dans l'alimentation, la santé et dans les pratiques rituelles de la population. Une représentativité de chaque espèce sur les fiches de relevés a permis de déterminer les espèces fréquentes et celles rares. L'analyse floristique a montré que les espèces de la famille des Fabaceae et Combrétaceae sont dominantes. Par ailleurs, une prédominance des phanérophytes sur les autres types biologiques a été notée et pourrait s'expliquer par des conditions édaphiques plus favorables à une végétation forestière ou à savanes boisées.

(C) 2015 International Formulae Group. All rights reserved.

Mots clés: Ampelocissus, diversité floristique, distribution, usages, Tchad.

\section{INTRODUCTION}

$\mathrm{Au}$ Tchad, la majeure partie de la population $(78 \%)$ vit essentiellement de l'agriculture et de l'élevage (INSEED, 2009). Le Tchad est un pays essentiellement agropastoral. L'élevage, une activité de second rang après l'agriculture, est pratiqué de façon traditionnelle et concerne essentiellement les bœufs d'attelage, les petits ruminants, les porcs et la volaille.

L'agriculture occupe une place importante dans l'économie tchadienne. Les ressources naturelles du sous-sol telles que le pétrole ont permis au gouvernement tchadien de déployer des efforts pour améliorer les productions agricoles en accompagnant les agriculteurs par la mise à leur disposition du matériel agricole notamment des tracteurs. Il a également initié l'aménagement de périmètres pour les cultures irriguées. Cependant, le Tchad enregistre en ce moment une baisse de la production agricole due essentiellement à l'exode rural, ajouté à l'avancée du désert et au manque de financement conséquent de l'agriculture. En outre, il est plus vraisemblable que l'origine de cette réduction de la production agricole soit liée à la 
diminution de la fertilité des sols associée aux maladies phytosanitaires.

Les cultures vivrières les plus répandues sont Pennisetum americanum (L.) K. Schum., Sorghum bicolor (L.) Moench., Zea mays L., Vigna unguiculata (L.) Walp., Arachis hypogea L. et Voandzeia subterranea L. (DC.). Les cultures de rente sont Gossypium barbadense L., Sesamum indicum L., Oryza glaberrima Steud., Ipomoea batatas (L.) Lam., Colocasia esculenta. Les produits maraîchers comme Lycopersicum esculentum Mill., Brassica oleracea L., Capsicum frutescens L., Solanum melongena L. et Nicotiana tabacum L. sont exploités à but lucratif.

La cueillette concerne essentiellement les espèces ligneuses exploitées pour leurs fruits. Ce sont entre autres Vitellaria paradoxa, Parkia biglobosa, Detarium microcarpum, Adansonia digitata, Balanites aegyptiaca, Ampelocissus, Cissus, Ziziphus mauritiana, Phoenix dactylifera, Annona senegalensis, Lannea acida, Lannea barteri.

Les populations tchadiennes, pour la plupart rurales, exploitent les ressources végétales spontanées pour assurer leur sécurité alimentaire. Cette exploitation se fait avec une sagesse intuitive et/ou empirique remarquable, sans techniques adéquates permettant de garantir la durabilité et la conservation de ces espèces. Ces espèces non cultivées ne bénéficient d'aucune protection surtout lorsqu'elles se trouvent hors des aires protégées. Elles sont souvent sous-exploitées du fait de la méconnaissance de leurs valeurs nutritives.

Aucun progrès durable, dans quelque domaine que ce soit, n'est à espérer des sociétés humaines tant que celles-ci ne jouiront pas d'une disponibilité alimentaire tant en quantité qu'en qualité pour un équilibre nutritionnel.

Les vitacées sont des plantes des régions tropicales (Afrique occidentale, Afrique orientale, Afrique centrale) et des régions tempérées (Asie, Europe, Amérique). Elles croissent en régions à climat soudanien ou soudano-guinéen. Ses espèces émettent de nouvelles tiges avant le retour des pluies (Akiko et Wen, 2006).

La plupart des espèces de la famille des vitacées sont comestibles malgré la toxicité de certaines espèces comme le Cissus quadrangularis et l'utilisation d'autres telles que le Cyphostemma laza dans les pratiques rituelles (Descoings et al., 2014).

Le genre Ampelocissus comprend un ensemble d'espèces qui sont des plantes admirablement adaptées aux plateaux soudanais et arides. Elles possèdent des tubercules fusiformes et charnus enfoncés profondément dans le sol. Les fruits des Ampelocissus sont très appréciés par les bergers qui conduisent les animaux au pâturage et les paysans aux champs. Les différents organes ont des valeurs rituelles et pharmacologiques non négligeables.

Les espèces du genre Ampelocissus sont des espèces spontanées à usage alimentaire. Au moment où le gouvernement tchadien prône l'autosuffisance alimentaire et la réduction de la pauvreté, l'exploitation des fruits et des feuilles des espèces du genre Ampelocissus pourrait contribuer à diversifier les sources alimentaires de la population. En effet, les jeunes feuilles et les fruits sont comestibles par l'Homme. La valorisation des fruits dans la fabrication des boissons rafraîchissantes pourrait être une source d'opportunités commerciales. En médecine traditionnelle, les espèces du genre Ampelocissus jouent un rôle dans les soins de santé à travers ses différents organes. Les feuilles et les tiges sont très appréciées par les animaux et en particulier galactogènes pour les vaches. Les rhizomes, très farineux, mélangés avec le son de sorgho, permettent l'engraissement des bœufs.

C'est dans cette optique que ces études ont été entreprises avec pour objectifs d'évaluer la diversité spécifique des espèces du genre Ampelocissus et leurs usages.

\section{MATERIEL ET METHODES}

\section{Site de l'étude}

Le site de l'étude est Donia, une souspréfecture du Département de Nya Pendé dans 
la région du Logone Oriental. Elle compte moins de 20.000 habitants et est distante du chef-lieu de la région (Doba) de $63 \mathrm{~km}$. La ville de Donia comprend les quartiers suivants : Bendoh I, Bendoh II, Bekounan, Gogoro, Maïrom Karwa, Djodjeti et Dombogo. Les villages environnants de Donia sont entre autres Melom, Madana Kôh, Ngara I, Ngara Beminda, Gongtou, Betelndôh, Ngamankourou. Les coordonnées géographiques de la sous-préfecture de Donia sont : $\mathrm{N} \quad 8^{\circ} 24^{\prime}$ et $\mathrm{E} \quad 16^{\circ} \quad 26^{\prime}$. Elle est caractérisée par un climat de type soudanoguinéen avec une pluviométrie annuelle variant entre 800 et $1400 \mathrm{~mm}$.

Dans cette zone, la saison des pluies s'étend de fin mai au début octobre. Donia se trouve dans la zone soudanienne dont les formations savanicoles sont dominantes. La zone soudanienne représente $12 \%$ du territoire national (CNAR, 2001). Physionomiquement, les végétations de Donia se subdivisent en forêt claire, savane boisée, savane arborée, savane arbustive et savane herbeuse.

\section{Collecte des données botaniques}

Une prospection préalable a permis de se rendre compte que les sites de prédilection des espèces du genre Ampelocissus sont diversement répandus. En effet, ces espèces sont répandues autour des termitières, au bord des cours d'eau, sur des rochers et dans les galeries forestières. Une appréciation visuelle a permis d'estimer le pourcentage de répartition de l'espèce dans les différents sites d'occurrence. Ainsi, les plateaux d'inventaire d'une superficie de $900 \mathrm{~m}^{2}$ chacun, ont été installés dans les différents sites de prédilection. La superficie de $900 \mathrm{~m}^{2} \mathrm{a}$ été retenue en tenant compte de l'aire minimale généralement adoptée en zone de savane et de certaines contraintes de visibilité dans les formations savanicoles (Amadé, 2006; Thiombiano, 2005).

La prospection a consisté à parcourir le site de l'étude afin de s'imprégner des réalités de terrain et d'identifier les différents sites de prédilection du genre Ampelocissus pour avoir une idée de la répartition du matériel à étudier. Cette mission a également permis de prendre contact avec les autorités locales pour leur présenter les objectifs de l'étude.

Pour les inventaires, les fiches de relevé phytosociologiques sont utilisées. Ces fiches portent les informations d'ordre général à savoir la date, l'auteur du relevé, la localité, les coordonnées GPS (Global Positioning System), la texture du sol, le type de formation, le taux de recouvrement des ligneux et leur hauteur moyenne. La texture du sol a été appréciée au toucher en prélevant la partie superficielle dont la profondeur varie entre $3-5 \mathrm{~cm}$. Les différentes hauteurs des individus ligneux ont été appréciées à l'aide d'une perche graduée. Les relevés ont consisté à recenser toutes les espèces ligneuses ou sous-ligneuses. Les espèces sont affectées d'un coefficient d'abondance dominance de Braun-Blanquet (1932). Ces coefficients sont définis comme suit :

$5=75$ à $100 \%$ de recouvrement;

$4=50$ à $75 \%$ de recouvrement;

$3=25$ à $50 \%$ de recouvrement;

$2=5$ à $25 \%$ de recouvrement;

$1=1$ à $5 \%$ de recouvrement;

$+=$ moins de $1 \%$ de recouvrement.

La plupart des relevés ont été réalisés dans les formations végétales à forêt-claire, savanes boisées et arborées.

Le matériel biologique est constitué de rameaux feuillés avec fleurs et/ou fruits des espèces ligneuses rencontrées dans les placettes. Les échantillons d'herbier de certaines espèces ligneuses du genre Ampelocissus sont récoltés à l'aide d'un sécateur. Les herbiers confectionnés sont identifiés ou confirmés à l'aide des spécimen de référence de l'herbier de laboratoire de Biologie et Ecologie Végétales de l'Université de Ouagadougou (Thiombiano et al., 2012 ) et de l'herbier National et Zootechnique de Ndjamena. Les inventaires se sont déroulés du 26 au 30 septembre 2013 et du 28 juin au 10 juillet 2014. 
Outils de collecte des données ethnobotaniques

Les enquêtes ethnobotaniques sont réalisées à travers une fiche élaborée en concertation avec des personnes ressources connaissant l'espèce Ampelocissus multistriata. Les principales informations recueillies sont l'identité de l'enquêté, les principaux usages de différents organes de l'espèce, la date, le lieu. Sur la base du contenu de la fiche d'enquête, des critères d'échantillonnage ont été définis aussi bien pour les enquêtés que les enquêteurs. En effet, le principal critère des enquêtés était l'âge (entre 35 et 80 ans car ces derniers connaissent mieux l'espèce). Pour les enquêteurs, il faut être de la localité et avoir un niveau d'instruction acceptable. L'échantillon est constitué de cinq personnes choisies du village et/ou quartier sachant lire et écrire le français. Les interviews sont réalisées sous forme de dialogue avec des personnes répondant aux critères.

\section{Traitement des données}

Les données phytosociologiques ont été traitées sur la base de la présence absence des espèces. Le tableur Excel 2007 a également servi pour la saisie et l'analyse des données.

\section{Détermination des espèces fréquentes et richesse floristique}

La richesse floristique et la fréquence des espèces ont été déterminées à partir de fiches des relevés. Pour la détermination des espèces fréquentes, une estimation a été faite par rapport au taux de représentativité de chaque espèce de toutes les familles rencontrées dans les relevés floristiques. Ainsi,

- les espèces fréquentes sont recensées dans au moins $50 \%$ de relevés ;

- les espèces peu fréquentes sont présentes dans 25 à $49 \%$ de relevés ;

- les espèces rares sont présentes dans moins de $25 \%$ de relevés.

En plus, les familles, les types biologiques et l'affinité phytogéographique de chaque espèce sont illustrés à l'aide des spectres.

Les types biologiques (TB) utilisés sont ceux définis par Raunkiaer (1905) et aménagés pour l'étude des formations végétales tropicales par divers auteurs (Lebrun, 1947 ; Lebrun, 1960 ; Kœchlin, 1961; Guillaume, 1967 ; Morat, 1973; Schnell, 1971; Boudouresque, 1995). Ce sont les Phanérophytes $(\mathrm{Ph})$, les chaméphyte $(\mathrm{Ch})$, les hémicryptophytes (He), les géophytes (Ge).

Les phanérophytes sont subdivisés en :

- mégaphanérophytes (MPh) : arbres de plus de 30 m de haut ;

- mésophanérophytes (Mph) : arbres de 10 à 30 m de haut;

- microphanérophytes (mph) : arbustes de 2 à $10 \mathrm{~m}$ de haut ;

- nanophanérophyes (nph) : sous-arbustes de moins de 2 m de haut;

Les types phytogéographiques (TP) utilisés sont basés sur les grandes subdivisions chorologiques établies pour l'Afrique (White, 1986) dont les principaux sont:

* espèces à large distribution qui regroupent:

- Cosmopolites $(\mathrm{Cos})$ = espèces largement répandues à la surface du globe ;

- Pantropicales $(\operatorname{Pan})=$ espèces réparties dans toutes les régions tropicales;

- Paléotropicales $(\mathrm{Pal})=$ espèces présentes aussi bien en Afrique tropicale, en Asie tropicale, en Australie et à Madagascar;

- Afro-américaines (AA) = espèces présentes en Afrique et en Amérique tropicale;

* espèces pluri-régionales africaines qui renferment:

- Soudano-zambésiennes $(\mathrm{SZ})$ = espèces présentes à la fois dans les Centres Régionaux d'Endémisme Soudanien et Zambésien;

- Afro-tropicales (AT) = espèces distribuées dans toute l'Afrique tropicale;

- Afro-malgaches (AM) = espèces distribuées en Afrique et à Madagascar ;

- Plurirégionales africaines $(\mathrm{PA})=$ espèces dont l'aire de distribution s'étend à plusieurs Centres Régionaux d'Endémisme ; 
- Guinéo-congolaises (GC) = espèces largement distribuées dans la Région guinéo-congolaise ;

- Soudano-guinéennes (SG) = espèces présentes à la fois dans les Centres Régionaux d'Endémisme Soudanien et guinéo-congolais ;

- élément-base qui regroupe essentiellement les espèces largement distribuées dans le Centre Régional d'Endémisme Soudanien (S).

\section{RESULTATS \\ Ecologie de Ampelocissus multistriata}

Les termitières mortes répandues dans la forêt claire de Donia constituent les zones de préférence $(70 \%)$ de Ampelocissus multistriata. Cette espèce se rencontre également le long de la rivière Nya, sur des sols humides (50\%). Par ailleurs, elle est présente sur les sols à faciès latéritiquescaillouteux du village Melom (15\%). Cette espèce se développe aussi dans les galeries forestières $(35 \%)$ du village Ngara Beminda (Figure 2).

\section{Identification des taxons}

L'identification des taxons prélevés a permis de noter principalement une espèce du genre Ampelocissus dénommée Ampelocissus multistriata (Baker) Planch. Des espèces du genre Cissus de la même famille ont également été identifiées parmi les taxons.

\section{Résultats de l'enquête ethnobotanique}

Les résultats de l'enquête basés sur deux cents (200) personnes interrogées sont consignés dans le Tableau1.Dans ce tableau, il ressort que tous les organes de Ampelocissus multistriata interviennent soit dans l'alimentation, soit dans les soins traditionnels, soit dans les croyances culturelles.

Par rapport aux feuilles, 48,5\% des personnes interrogées ont indiqué que les feuilles de Ampelocissus multistriata ont un intérêt médical important. La totalité des personnes consultées trouvent que les feuilles constituent le fourrage pour les animaux. Par contre, $6,25 \%$ autres ont trouvé leur importance dans les croyances culturelles.

En ce qui concerne les fruits, $100 \%$ des personnes trouvent que les fruits sont comestibles. Vingt-cinq (25)\% autres trouvent que les fruits ont un intérêt médicinal non négligeable alors qu'aucune personne n'a indiqué l'intérêt culturel des fruits.

Quant aux écorces, 33\% des personnes interviewées trouvent que celles-ci entrent dans les soins médicaux traditionnels. Cinq (5)\% d'entre elles ont évoqué l'importance culturelle et traditionnelle alors qu'aucune personne n'a signalé leur intérêt alimentaire.

Pour les racines tubéreuses, $37,5 \%$ des personnes interrogées trouvent que celles-ci interviennent dans les soins médicinaux. Soixante-dix (70)\% autres trouvent que ces racines tubéreuses servent à l'engraissement des bœufs et ont un rôle galactogène. Enfin, $18,75 \%$ ont précisé les rôles que jouent les racines dans les croyances culturelles.

Les moyennes de pourcentage des personnes interrogées en fonction des usages sont les suivantes: l'usage médicinal représente un pourcentage de $37,5 \%$; l'usage culturel $31,7 \%$ et l'usage alimentaire $10,12 \%$.

\section{Richesse floristique \\ Spectre de familles}

Au total, 110 relevés ont été effectués au cours des investigations phytosociologiques. Quatre-vingt-six (86) espèces réparties dans 61 genres et 29 familles ont été recensées (Thiombiano et al., 2012). En effet, les familles les plus représentées sont les Fabaceae $(25,58 \%)$ et les Combretaceae $(12,79 \%)$. Elles sont suivies de très loin par les Rubiaceae (6,97\%), Euphorbiaceae $(5,81 \%)$ et Tiliaceae $(4,65 \%)$. Cinq familles ont chacune un pourcentage de $3,48 \%$. Il s'agit des Vitaceae, Moraceae, Anacardiaceae, Chrysobalanaceae et Rhamnaceae. Enfin, quatre familles ont chacune un pourcentage de $2,32 \%$. Ce sont les familles des Loganiaceae, Annonaceae, 
Appocynaceae et Verbenaceae). Quinze (15) familles sont regroupées sous «Autres familles» car possédant moins de 2 espèces chacune et représentant $17,44 \%$ de l'ensemble des familles rencontrées (Figure 3).

Spectre de types biologiques

Les relevés phytosociologiques étant réalisés uniquement sur des espèces végétales ligneuses, les types biologiques concernent beaucoup plus les phanérophytes. C'est ainsi qu'il ressort une large dominance des phanérophytes $(97,68 \%)$ sur les chaméphytes $(2,32 \%)$ (Figure $4 a)$.

Une analyse au sein des phanérophytes a révélé une nette dominance des microphanérophytes $\quad(59,5 \%) \quad$ sur lesmésophanérophytes $(26,2 \%)$ et sur les phanérophytes lianescents $(9,5 \%)$. Les nanophanérophytes $(3,6 \%)$ et les mégaphanérophytes $(1,2 \%)$ sont très peu représentés (Figure4b).

\section{Affinités phytogéographiques}

Le spectre phytogéographique global (Figure 5) présente une forte proportion de l'élément-base soudanien $(31,76 \%)$, suivi des espèces soudano-zambéziennes $(25,88 \%)$ et des afro-tropicales $(15,9 \%)$. Si les paléotropicales (Pal) et les autres espèces plurirégionales (PA) sont faiblement représentées, alors les pantropicales (Pan), les afromalgaches (AM), les soudano-guinéennes $(\mathrm{SG})$ et les espèces guinéennes $(\mathrm{G})$ sont moins importantes. Aussi, les espèces guinéocongolaises (GC) et les afro-américaines sont absentes.

\section{Espèces fréquentes}

La Figure 6 montre que la plupart des espèces, au nombre de 40, sont rares car elles sont dans moins de $25 \%$ de relevés.

Vingt-trois (23) espèces représentant un pourcentage de $26,7 \%$ sont peu fréquentes. Ce sont entre autres Crossopteryx febrifuga, Bridelia scleroneura, Combretum glutinosum,
Commiphora pedunculata, Erythrophleum suaveolens, Grewia cissoides, Grewia venusta, Securidaca longipedunculata, Strychnos innocua, Vitex madiensis, Terminalia macroptera et Vitellaria paradoxa. Ces espèces sont présentes dans au moins $42 \%$ de relevés. Les autres espèces peu fréquentes représentant un pourcentage de $34,54 \%$ sont Monotes kerstingii (33,63\%), Grewia flavescens (32,72\%), Hexalobus monopetalus $(32,72 \%)$, Pericopsis laxiflora $(31,81 \%)$, Sterculia setigera (30\%), Cassia sieberiana (28,18\%), Vitex doniana $(26,36 \%)$, Grewia bicolor (25,45\%), Gardenia aqualla (24,54\%), Lonchocarpus laxiflorus (24,54\%), Ximenia americana (24,54\%), Maytenus senegalensis (20\%), Terminalia mollis (18,18\%), Khaya senegalensis (15,45\%), Diospyros mespiliformis (10\%), Guiera senegalensis $(10 \%)$.

On note également la présence de vingt-trois (23) espèces estimées comme étant des espèces fréquentes et représentant un pourcentage de $27,6 \%$. Ces espèces se trouvent dans au moins $50 \%$ des relevés. Dans ce groupe, on peut distinguer des espèces très fréquentes car elles sont présentes dans (75\%) de relevés. Ce sont: Allophylus africanus, Annona senegalensis, Burkea africana, Cissus tuberosa, Cissus rufescens, Detarium microcarpum, Entada africana, Flugea virosa, Hymenocardia acida, Piliostigma thonningii, Prosopis africana, Stereospermum kunthianum, Strychnos spinosa et Terminalia laxiflora. Les autres espèces fréquentes sont Pterocarpus lucens (66,36\%), Daniellia oliveri (66,36\%), Lannea barteri (62,72\%), Combretum collinum $(60,90 \%)$, Bridelia ferruginea (59,09\%), Combretum molle (58,18\%), Anogeissus leiocarpus (57,27\%), Cissus populnea (56,36\%), Combretum nigricans $(56,36 \%)$, Dichrostachys cinerea $(51,81 \%)$. 


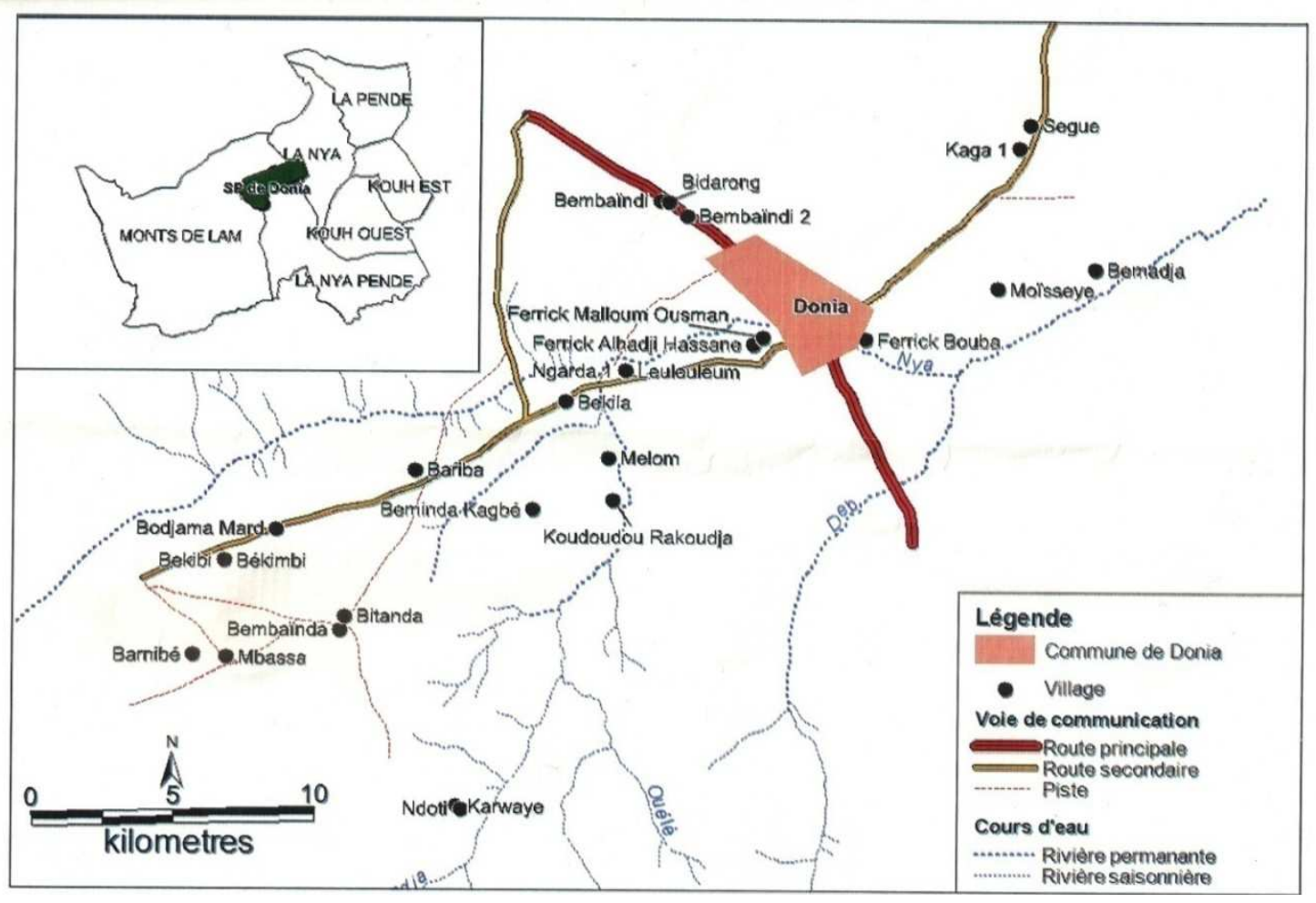

Figure 1: Localisation de la zone de l'étude (Ratna, 2012).

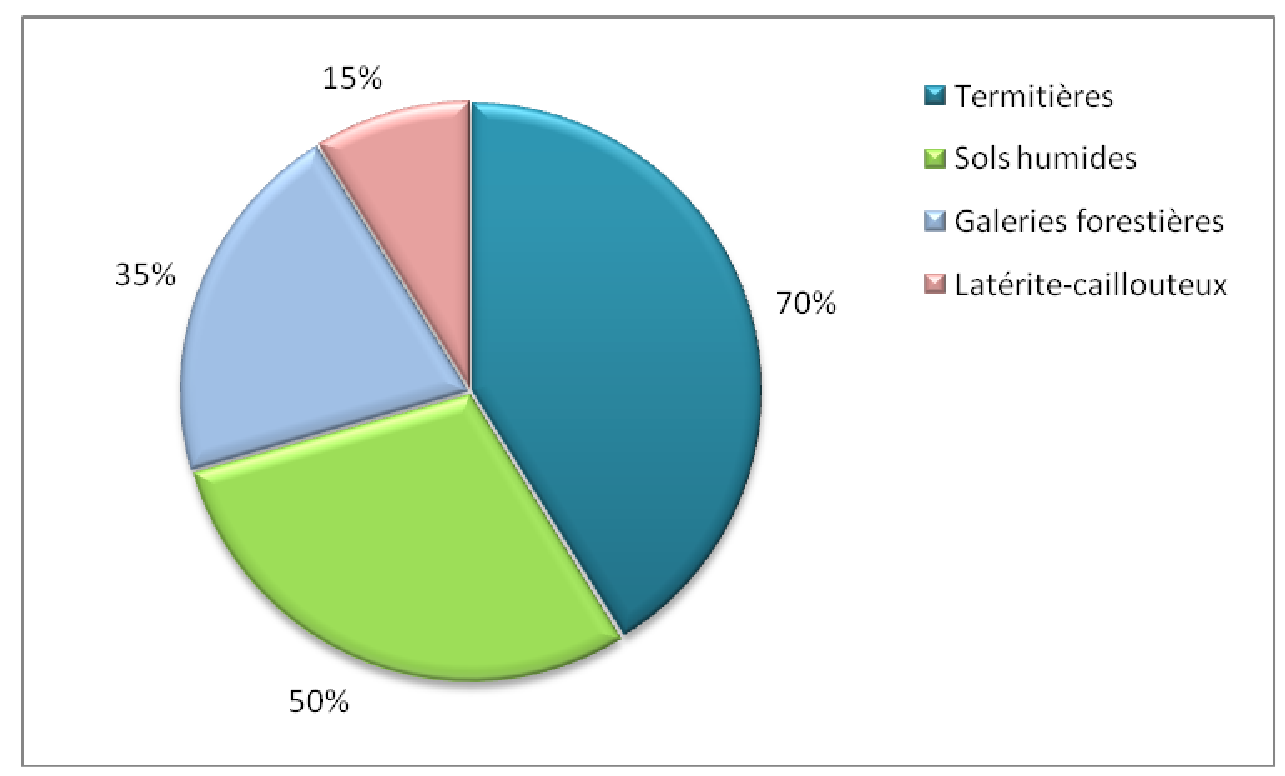

Figure 2: Répartition de Ampelocissus multistriata en fonction de différents types de sols. 


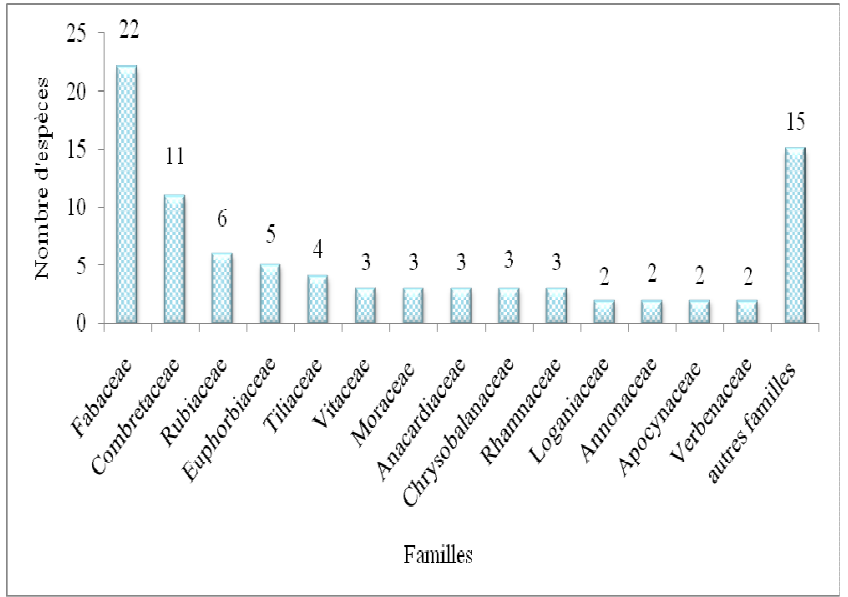

Figure 3: Spectre des familles des espèces ligneuses.

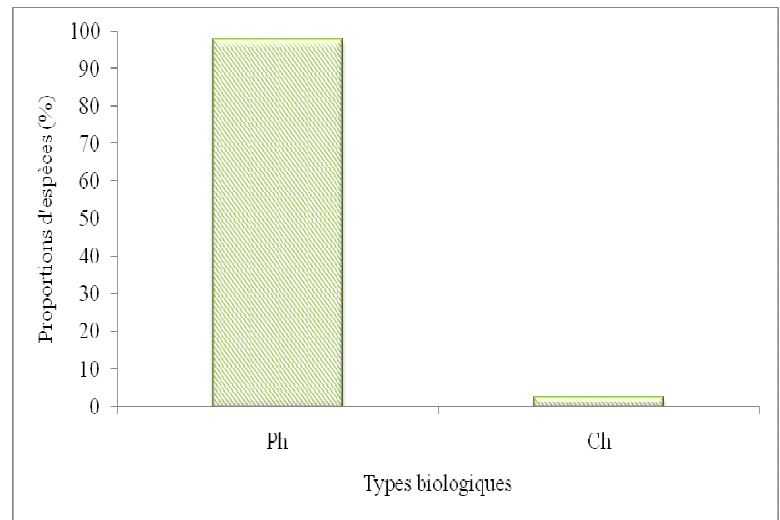

Figure 4a: Spectre de types biologiques. Ph : phanérophytes; Ch : chaméphytes.

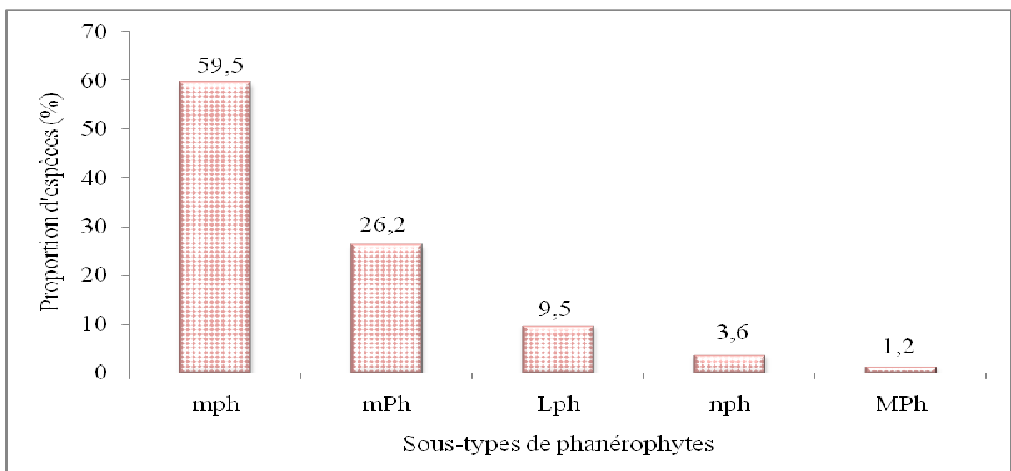

Figure 4b: Spectre de sous-types de phanérophytes. mph: microphanérophytes ; nph : nanophanérophytes ; mPh : mésophanérophytes; MPh : mégaphanérophytes ; Lph : phanérophytes lianescents. 


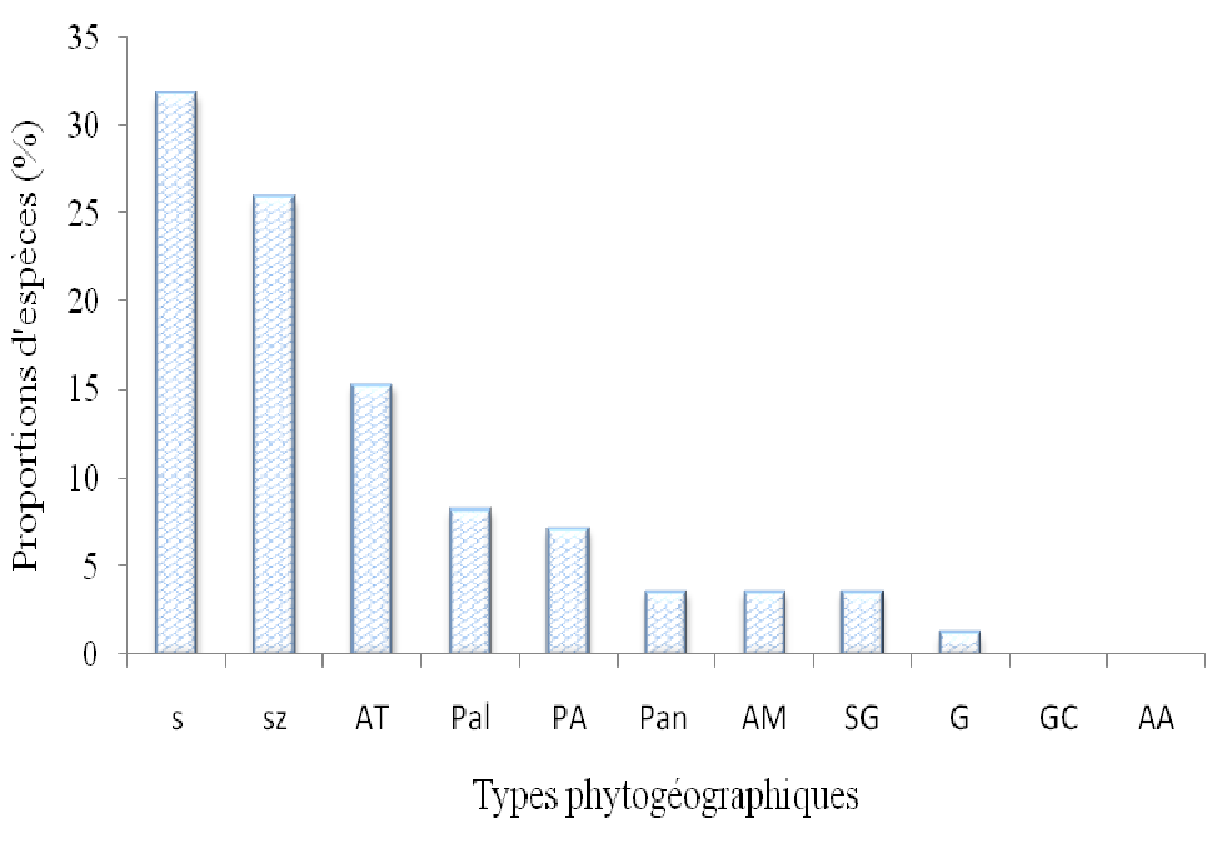

Figure 5: Spectre de types phytogéographiques. S : élément-base soudanien ; SZ : espèces soudanozambéziennes; Pan: espèces pantropicales ; Pal: espèces Paléotropicales ; AT: espèces afro-tropicales ; PA: autres espèces pluri-régionales africaines ; AM : espèces afro-malgaches ; GC: espèces guinéo-congolaises ; SG : espèces soudanoguinéennes ; AA : espèces afro-américaines, $\mathrm{G}$ : espèces guinéennes.

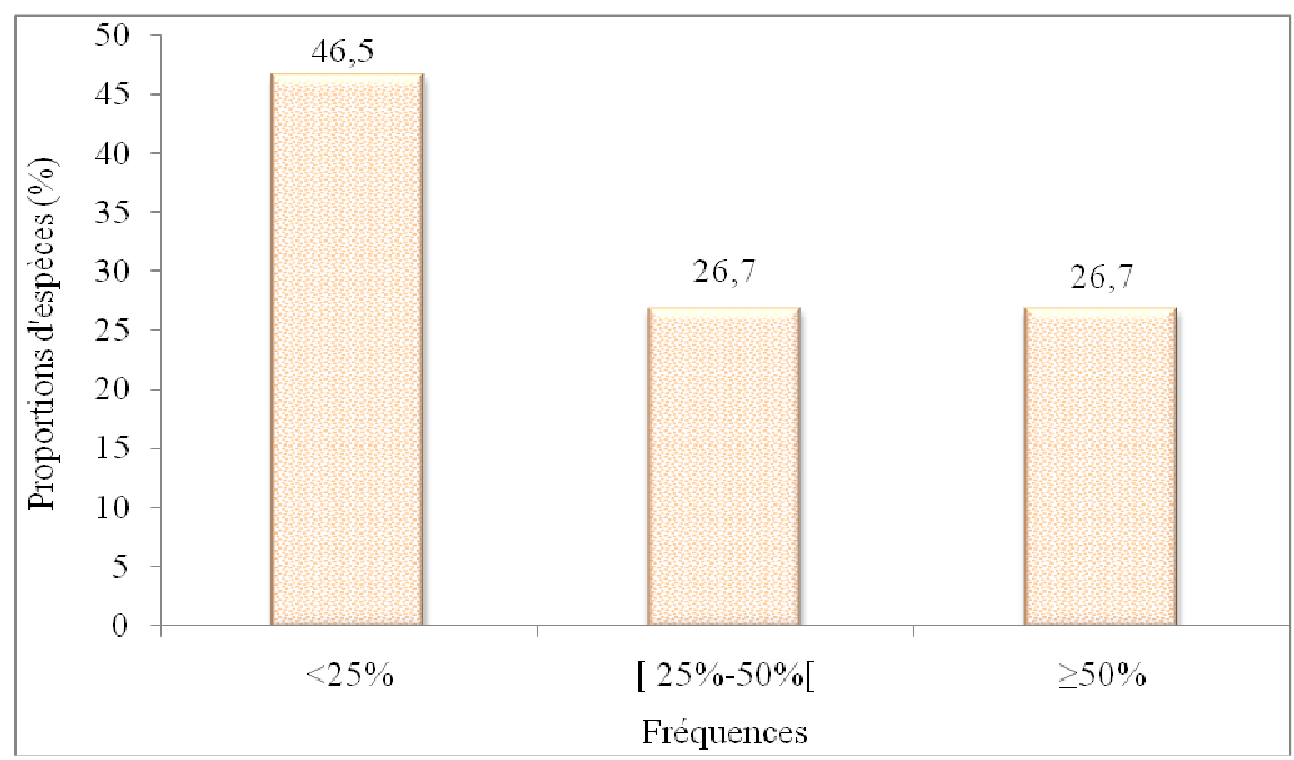

Figure 6 : Spectre de fréquences spécifiques. 
Tableau 1 : Différents usages des organes de Ampelocissus multistriata.

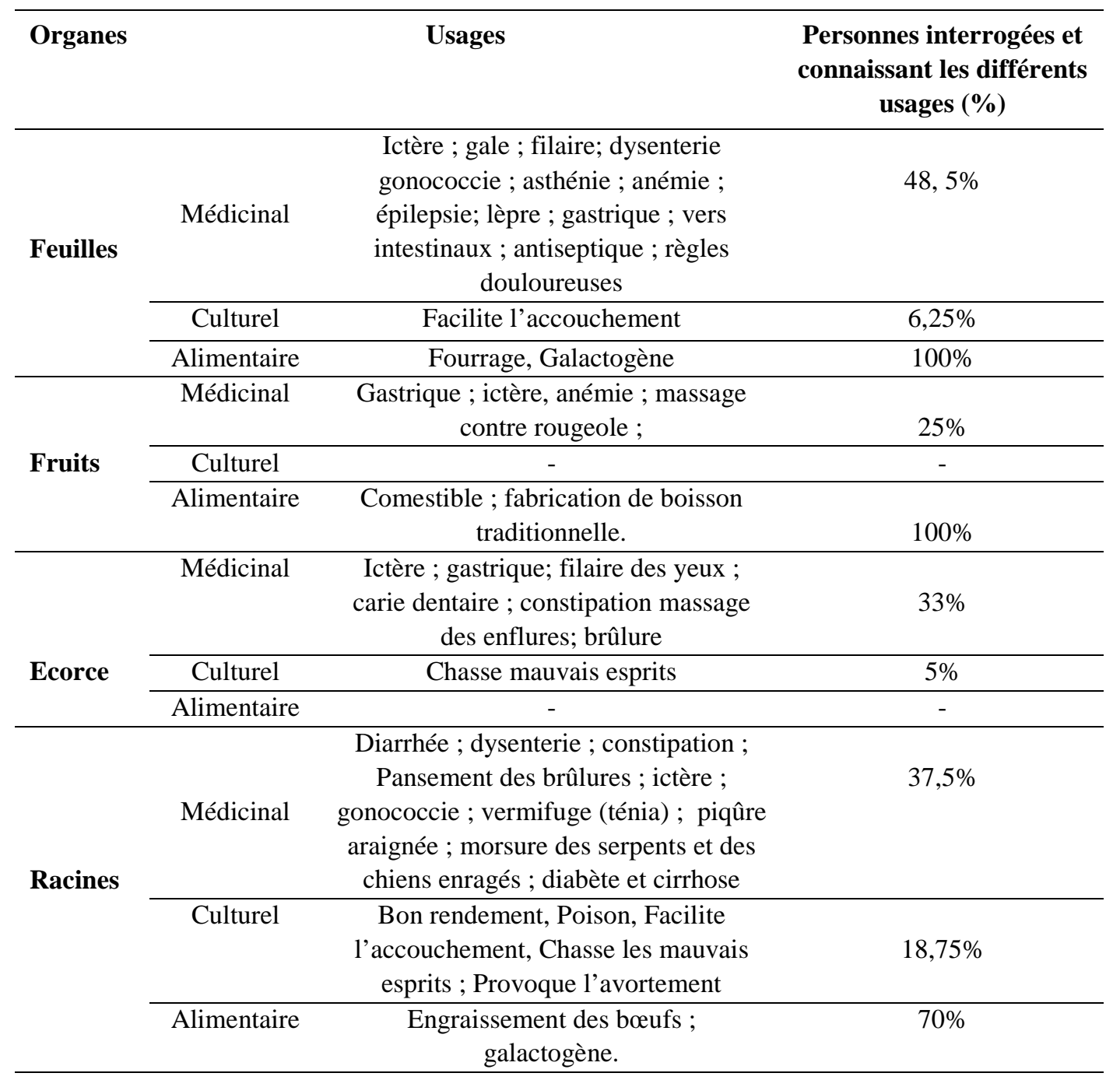

\section{DISCUSSION}

Les résultats de l'étude révèlent que Ampelocissus multistriata est une espèce qui se développe sur divers types de sols. Les prospections effectuées ont permis d'observer l'abondance de cette espèce autour des termitières. Aussi, la trouve-t-on au bord de la rivière Nya, sur des sols humides, dans les galeries forestières de Ngara Beminda. Thiombiano et al. (2006) ont récolté cette espèce dans la forêt classée de Niangoloko, dans la mare aux hippopotames de Bala au Burkina Faso. Adam et al. (2014) l'ont récoltée dans les galeries forestières au Sénégal. Cette plante préfererait une certaine humidité pour son développement. C'est pourquoi, elle se retrouve dans la souspréfecture de Donia, située dans la partie du territoire qui est la mieux arrosée du pays. Ceci pourrait expliquer l'aire de distribution de la plante qui serait strictement sudsoudanienne. La plante est rencontrée en 
faible proportion sur les sols à faciès latéritiques-caillouteux. C'est le cas de village Melom à l'ouest de Donia. Wala (2004) a rapporté que cette espèce évolue sur des montagnes et rochers. Aussi, Zongo (2012) a récolté cette plante dans la commune de Saponé à environ $45 \mathrm{~km}$ de Ouagadougou. Ainsi, selon Thiombiano (2005), la distribution des espèces constitue un outil indispensable dans la connaissance de l'écologie des espèces.

L'inventaire a permis de trouver une seule espèce du genre qui est Ampelocissus multistriata (Baker) Planch. Descoings (1975), en travaillant au sud du Tchad, a trouvé 3 espèces de ce genre. Ces espèces sont: Ampelocissus africana (Loureiro) Merrill, Ampelocissus sarcocephala (Schw.) Planch. Et Ampelocissus pentaphylla (Guill et Perr.) Gilg. qui est une espèce synonyme de Ampelocissus multistriata (Baker) Planch. Cette différence pourrait s'expliquer par l'étendue du territoire parcouru par Descoings qui est beaucoup plus vaste que la nôtre. Par contre, plusieurs espèces du genre Cissus de la même famille ont été rencontrées sur le site de l'étude. Adomou (2005) a signalé dans ses travaux que le genre Cissus est bien représenté dans les savanes soudaniennes.

Des résultats de l'enquête,il ressort que les racines et les feuilles sont utilisées pour faire le pansement des plaies et comme vermifuges. Bah et al. (2006) avaient souligné que les jeunes feuilles sont appliquées en emplâtre sur les abcès et les bubons (tumeurs inflammatoires liées aux glandes lymphatiques). Ainsi donc, la plante aurait des propriétés anthelminthiques et antiseptiques.

En outre, la décoction des racines calme les douleurs musculaires et articulaires. Ces résultats sont en conformité avec ceux de Nadembega et al. (2010) qui avaient souligné que les racines de Ampelocissus multistriata ont des effets sédatifs. Ce résultat a également été confirmé par Arbonnier (2009) qui considère les racines de cette espèce comme un véritable anti-inflammatoire. Les enquêtes ont aussi révélé que tous les organes de cette plante entrent dans l'alimentation. Les tiges et les feuilles sont utilisées comme fourrage et très appréciées par les animaux. Ces résultats sont conformes à ceux de Arbonnier (2009) qui avait mentionné les valeurs nutritives de cette plante. Les fruits sont comestibles et ont un goût vraiment agréable. Les enquêtes ont également révélé que Ampelocissus multistriata est utilisé dans les pratiques culturelles de la population de Donia. En effet, les racines de ces plantes, réduites en poudre, mélangées avec la semence, permettent d'avoir une bonne production lors de récolte. Aussi, la racine de cette plante est utilisée pour faciliter l'accouchement des femmes. Cette opinion est partagée par Arbonnier (2009) qui a largement évoqué le caractère culturel de cette plante.

La moitié des espèces répertoriées sont peu fréquentes. Cette rareté de certaines de ces espèces est due aux exigences des conditions écologiques. Pour d'autres, elle serait due aux activités anthropiques et aux pressions de pâturage qui ont conduit à leur rareté.

D'après l'analyse floristique, le spectre de familles montre une importance des Fabaceae et Combrétaceae sur le site de l'étude dominé par les formations savanicoles et la forêt claire. Ceci peut être illustré par l'opinion de Thiombiano (2005) qui avait rapporté que les Combretaceae sont abondamment représentées dans les savanes et quelques forêts denses humides de l'Afrique intertropicale. Cet auteur a également souligné que la quasi-totalité des espèces des Combretaceae trouvent leur optimum écologique dans la zone beaucoup plus soudanienne. 
Les résultats ont montré que les phanérophytes, avec un spectre de 97,68\%, sont les types biologiques les plus abondants. Cette abondance élevée est l'un des indicateurs de bonnes conditions écologiques stationnelles au sein de ces formations savanicoles et ces forêts claires humides. Ainsi, la prédominance des phanérophytes traduirait des conditions édaphiques plus favorables à une végétation forestière ou encore à savanes boisées. Ouoba (2006) a aussi montré que dans les zones à climat tropical humide, il y a une nette dominance des phanérophytes sur les autres formes de types biologiques marquant ainsi le caractère forestier de la végétation. La forte représentativité des phanérophytes exprimerait une grande richesse spécifique de la flore boisée. Pour Niang-Diop et al. (2010), les types biologiques désignent le comportement adaptatif de l'espèce. Ils renseignent sur le type de formation végétale, son origine et ses transformations. C'est pourquoi Mbayngone (2008) a trouvé que les proportions non négligeables des mésophanérophytes $(26,2 \%)$ et dans une moindre mesure des microphanérophytes pouvant induire une structure arborescente. En effet, les microphanérophytes sont des phanérophytes représentés par des arbustes de 2 à $10 \mathrm{~m}$ de hauteur. Par rapport aux résultats obtenus, ce nombre élevé des phanérophytes est dû à une forte importance des microphanérophytes.

Il est à noter l'importance des espèces de l'élément-base soudanien et des espèces soudano-zambéziennes sur les autres types phytogéographiques. Ce qui montre cependant que les espèces soudaniennes et les espèces soudano-zambéziennes sont bien représentées sur le site de l'étude. Cette situation résulterait de la position méridionale du site et de l'existence d'un régime montagneux (village Melom) créant un micro-climat favorable à l'installation des espèces soudano-guinéennes.
De façon générale, la prédominance des espèces de l'élément-base soudanien et des espèces soudano-zambéziennes sur les autres types phytogéographiques est une caractéristique des savanes soudaniennes (Pallo et Sawadogo, 2010; Houinato, 2001; Adomou, 2005).

\section{Conclusion}

Cette étude a permis d'une part, de faire l'inventaire des espèces ligneuses, de déterminer l'espèce Ampelocissus multistriata et son écologie dans le site de l'étude et d'autre part, de connaître les différents usages des organes de cette espèce à travers des enquêtes. Les relevés effectués au niveau des forêts claires et des savanes de Donia, ont permis de comprendre qu'elles sont composées de 86 espèces ligneuses réparties en 61 genres et 29 familles. Les résultats ont montré que les fruits de Ampelocissus multistriata sont comestibles par l'Homme et certains animaux tels que le singe et les oiseaux. Les tiges et les feuilles sont utilisées comme fourrage pour les animaux. Les racines, tiges, feuilles et écorces de cette plante interviennent efficacement dans les soins traditionnels et dans les activités culturelles de la population de Donia.

Les prospections effectuées ont révélé que Ampelocissus multistriata évolue sur divers types de sol. Les relevés floristiques ont permis de déterminer les espèces fréquentes, peu fréquentes et celles qui sont rares sur ce site. Les familles les plus représentées sont les Fabaceae et Combretaceae.

$$
\text { L'analyse générale des types }
$$
biologiques montre que les microphanérophytes sont plus représentés que les autres, illustrant ainsi le caractère forestier de cette zone. Aussi, les espèces de l'élémentbase soudanien et les espèces soudano- 
zambéziennes dominent sur les autres types phytogéographiques.

\section{REMERCIEMENTS}

Les auteurs remercient l'Université de Ouagadougou, l'Université de Ndjamena, le Laboratoire Info Bio de l'Université de Ouagadougou pour tous les efforts consentis dans la réalisation de cette étude. Ils remercient par ailleurs Campus France pour le financement de ce travail.

\section{REFERENCES}

Adam J, Taylor F. 2014. Note sur la végétation des Niayes de la presqu'Île du Cap Vert (Dakar A.O.F). Bulletin de la Société Botanique de France, 100: 4-6.

Adomou AC. 2005. Vegetation patterns and environnemental gradients in Benin.Implications for biogeography and conservation. $\mathrm{PhD}$ thesis, Wageningen University, p. 132.

Akiko S, Wen J. 2006. Philogenetic analysis of the grape family (Vitaceae) based on three chloroplast markers. American Journal of Botany, 93(2): 278-287.

Amadé O. 2006. Diversité et dynamique de la végétation ligneuse de la partie orientale du Burkina Faso. Thèse de doctorat, Université de Ouagadougou, p.196.

Arbonnier M. 2009. Arbres, Arbustes et Lianes des Zones Sèches d'Afrique de l'Ouest ( $\left.3^{\mathrm{e}} \mathrm{edn}\right)$. Le Quae; 576.

Bah S, Diallo D, Dembele S, Paulsen BS. 2006. Ethnopharmacological survey of plants used for the treatment of schistosomiasis in Niono District, Mali. $J$. Ethnopharmacol., 105: 387-399.

Braun-Blanquet J. 1932. Plant sociology. The study of plant communities (Ed. Mc Gray, Mc Hill): New York: London; 439.

Boudouresque CF. 1995. Critères de sélection et projet de liste des espèces en danger ou menacées. Tunisie ; 1-33.
CNAR. 2001. Carte bioclimatique. Centre National d'Appui à la Recherche.

Descoings B, Taylor F. 2014. Cyphostemma (Vitacée) nouveaux de Madagascar. Bulletin de la Société Botanique de France, 9(109): 226-276.

Descoings B. 1975. Les Vitacées du Tchad. Adansonia, ser.2, 14(4): 655-680.

Guillaumet JL. 1967. Recherches sur la végétation et la flore de la région du BasCavally (Côte d'Ivoire). Mémoires O.R.S.T.O.M. $\mathrm{n}^{\circ}$ 20, paris, p.249.

Houinato MRB. 2001. Phytosociologie, écologie, production et capacité de charge des formations végétales pâturées dans la région des Monts Kouffé (Bénin). Thèse de l'Université Libre de Bruxelles, p. 218.

INSSEED. 2009. Recensement General de la Population et de l'Habitat au Tchad. Institut National des Etudes Economiques et Démographiques.

Koechlin J. 1961. La végétation des savanes dans le sud de la République du Congo Brazzaville. Edition Imprimerie Charité : Montpellier, France ; 310.

Lebrun J. 1947. La Végétation de la plaine alluviale au sud lac Edouard. Fascicules 2(1), Inst. des Parcs Nat. du Congo Belge, Bruxelles, p. 800.

Lebrun J. 1960. Sur une méthode de délimitation des horizons et étages de végétation des montagnes du Congo oriental. Bull. Jard. Bot. Etat, 30: 75-94.

Mbayngone E.2008.Flore et végétation de la réserve partielle de faune de Pama, SudOuest de Burkina Faso. Thèse unique, Univ. Ouaga., p.137.

Morat P. 1973. Les savanes du Sud-ouest de Madagascar. Mémoire ORSTOM. $\mathrm{N}^{\circ} 68$, paris, p. 235.

Nadembega P., Boussim J.I., Nikiema J.B., Poli F., Antognoni F. 2010. Medicinal plants in Baskoure, Kourittenga Province; 
Burkina Faso: an ethnobotanical study. $J$. Ethnopharmacol., 133(2): 378-395.

Niang-Diop Sambou F, Lykke AM. 2010. Contraintes de régénération naturelle de Prosopis africana : facteurs affectant la germination des graines. Int. J. Biol. Chem. Sci., 4(5): 1693-1705.

Ouoba P. 2006. Flore et Végétation de la forêt classée de Niangoloko, sud-ouest du Burkina Faso. Thèse unique, Univ. Ouaga., p.144.

Pallo FJP, Sawadogo N. 2010. Essai de corrélation entre les caractéristiques du climat, de la végétation et des sols au Burkina Faso. Int. J. Biol. Chem. Sci., 4(5):1839-1850.

Raunkiaer C. 1905. Types biologiques pour la géographie botanique. Bulletin Academy of Royal Science, 5: 347-437.

Schnell R. 1971. Introduction à la phytogéographie des pays tropicaux. Les milieux, les groupements végétaux. Gauthier-Villars, Paris, 3(4): 503-951.

Thiombiano A. 2005. Les Combretaceae du Burkina Faso : taxonomie, écologie, dynamique et régénération des espèces.
Thèse de Doctorat d'Etat, Université de Ouagadougou, p. 290.

Thiombiano A. 2006. Laboratoire Info bio (Herbier) de l'Université de Ouagadougou.

Thiombiano A, Schmidt M, Dressler S, Amadé O, Hehn K, Zizka G. 2012. Catalogue des plantes vasculaires $\mathrm{du}$ Bukina Faso, p. 391.

Wala K. 2004. La végétation de la chaîne de l'Atakora au Bénin : Diversité floristique, phytosociologie et impact humain. Thèse de Doctorat, Université de Lomé, p.140.

White F. 1986. La végétation de l'Afrique. Mémoire accompagnant la carte UNESCO de végétation, p. 383.

Zongo C. 2012. Etude photochimique et pharmacologique de quelques plantes communément utilisées en Afrique de l'Ouest pour lutter contre les infections parasitaires et microbiennes. Thèse unique de l'Uni. de Ouaga., p. 178. 